Horizons philosophiques

\title{
Développement moral et jugement moral : réexamen de la controverse Kohlberg-Gilligan
}

\section{Nathalie Savard}

Volume 7, numéro 1, automne 1996

Le dernier pont

URI : https://id.erudit.org/iderudit/801033ar

DOI : https://doi.org/10.7202/801033ar

Aller au sommaire du numéro

Éditeur(s)

Collège Édouard-Montpetit

ISSN

1181-9227 (imprimé)

1920-2954 (numérique)

Découvrir la revue

Citer cet article

Savard, N. (1996). Développement moral et jugement moral : réexamen de la controverse Kohlberg-Gilligan. Horizons philosophiques, 7(1), 113-124.

https://doi.org/10.7202/801033ar d'utilisation que vous pouvez consulter en ligne.

https://apropos.erudit.org/fr/usagers/politique-dutilisation/ 


\section{DÉVELOPPEMENT MORAL ET JUGEMENT MORAL : RÉEXAMEN DE LA CONTROVERSE KOHLBERG-GILLIGAN*}

Une controverse concernantle développement moral oppose depuis plusieurs années les tenants de deux écoles de pensée. L'une se rattache à la position dissidente de Carol Gilligan, l'autre à la théorie de celui qui à l'origine fut son maître, Lawrence Kohlberg'. À la source de la controverse se trouve d'abord le fait que Kohlberg propose un modèle psychologique qui prétend que la capacité formelle de raisonner selon des principes de justice se développe chez tout individu, selon des stades invariables et universels, du moment qu'il est en relation avec autrui. Autrement dit, chaque individu développe cette capacité en suivant certaines étapes invariables, successives, irréversibles et ordonnées selon un modèle universel. Or, Kohlberg a cherché à mesurer ce développement, et par là à vérifier son modèle, en concevant un test portant sur la résolution de dilemmes moraux hypothétiques. Etc'està partir de l'interpré-

Le présent article est le fruit d'un travail effectué dans le cadre des activités du Groupe de recherche sur l'allocation des ressources rares en matière de transplantation d'organes dirigé par Madame Jocelyne Saint-Arnaud du Département des sciences religieuses de l'Université du Québec à Montréal. Ce groupe bénéficie d'une subvention du Conseil de recherche en sciences humaines du Canada pour la réalisation de son projet.

Je remercie les membres du groupe de recherche, particulièrement Mesdames Jocelyne Saint-Arnaud et Johanne Pomerleau, pour leurs commentaires et critiques stimulantes. Je remercie également Monsieur Pierre Gendron du Centre de recherche en droit public de l'Université de Montréal pour son aide précieuse lors de la révision du texte.

Une première version de ce texte été présentée le 26 novembre 1994 à Ottawa dans le cadre du sixième congrès annuel de la Société canadienne de bioéthique.

1. Les principaux ouvrages qui ont servi de base à la rédaction de cet article sont : Lawrence Kohlberg, The psychology of moral development - The nature and validity of moral stages (Essays on moral development, vol. II). San Francisco : Harper \& Row Publishers, 1984, et Carol Gilligan, In a different voice - psychological theory and women's development. Cambridge et Londres : Harvard university press, 32e édition, 1993 (en français : Une si grande différence (traduction de A. Kwiatek). Paris, Flammarion, 1986.) 
tation à donner à certains des résultats obtenus que Gilligan formulera une critique quant à la portée véritable de la théorie de Kohlberg et qu'elle proposera une alternative, d'où la controverse que l'on sait.

En examinant l'ensemble des résultats, Gilligan constate que certains faits résistent à la théorie et elle parvient à la conclusion que ce modèle n'est pas d'une portée aussi générale que Kohlberg le prétend. En effet, elle croit que le modèle proposé ne rend compte en fait que d'une orientation possible, parmi d'autres, face aux problèmes moraux que nous rencontrons dans notre vie quotidienne. Pour Gilligan, le modèle de Kohlberg n'est pas universel (au sens où il serait suivi et adopté par tous les individus). Elle parvient à cette conclusion en constatant que les femmes obtiennent systématiquement des scores plus bas que les hommes, ce qui les singularise comme groupe, ce fait n'étant pas expliqué de manière satisfaisante par Kohlberg. Or, à son avis, la résolution des dilemmes moraux ne consiste pas seulement en une application froide de principes de justice afin d'établir un équilibre entre les droits et revendications de chacun, ce qui caractériserait surtout la démarche masculine. Selon elle, d'autres motivations peuvent nous guider pour aborder une situation et y porter un jugement moral, notamment la sollicitude (caring) ${ }^{2}$, qui ressortirait davantage d'une démarche féminine. Et c'est cette approche que privilégie Gilligan et qu'elle développe à partir des résultats auxquels ses études (réalisées particulièrement auprès des femmes) lui ont permis de parvenir.

Sans doute les données empiriques relatives à la persistance «anormale» des faibles scores des femmes au test de Kohlberg considérées par Gilligan sèment-elles le doute quantà l'efficacité et à la portée réelle de la théorie de Kohlberg. Mais, de ces contre-exemples, on ne peut conclure que deux choses : ou bien la théorie est inadéquate, ou bien les outils empiriques mis

2. Le terme employé par Gilligan est "caring" ou "care". La traduction courante de ce terme est "sollicitude". Bien qu'à notre avis le terme français ne rende pas compte des nombreux sens associés, en anglais, aux termes "care" et "caring" (i.e. "attention", "soin", "se soucier de", "être attaché à", "s'intéresser à", etc.), nous conserverons néanmoins la traduction courante. 
au point pour tester la théorie sont mal conçus (en ce qu'ils ne mesurent pas ce qu'ils sont censés mesurer).

Nous sommes d'avis que c'est cette dernière conclusion qui est la plus plausible. Sa confirmation demande toutefois des connaissances de la méthodologie d'investigation en psychologie que nous ne possédons pas. Cette question ne pourra donc être réglée que par les psychologues.

Nous pensons cependant que, indépendamment de ces difficultés, les théories de Kohlberg et de Gilligan présentent un intérêt proprement philosophique. Aussi, malgré les divergences qui semblent les séparer, nous croyons que ces écoles de pensée partagent les mêmes présupposés philosophiques et que, de ce fait, elles ne sont pas incompatibles et peuvent même conjuguer leurs efforts à l'intérieur d'un même projet.

Cette démonstration portera sur la constatation que les théories respectives de Gilligan et de Kohlberg ne portent pas sur le même objet et s'inscrivent dans des domaines distincts de l'éthique. Cela étant, l'opposition n'aurait plus aucune raison d'être et la controverse deviendrait sans objet. Aussi, dans les pages qui suivent, nous établirons que la position développée par Gilligan relève du domaine normatif substantiel de la moralité et qu'elle s'oppose, de par sa nature, aux positions normatives substantielles qui réduisent la moralité aux considérations de justice. Or, la tendance à croire, à tort selon nous, que c'est là la démarche de Kohlberg a contribué à la naissance de la controverse. Nous sommes convaincus que le projet de Kohlberg s'inscrit non pas dans l'articulation du contenu de la moralité mais bien dans la réflexion sur la possibilité même de la moralité et de la connaissance morale. Le modèle formel du développement et de l'acquisition des compétences morales par stades structurels établit cette possibilité. En effet, son modèle n'est qu'un cadre structurel à l'intérieur duquel plusieurs positions normatives substantielles peuvent être articulées.

Kohlberg propose une théorie qui cherche à comprendre le développement du raisonnement de justice (justice reasoning). 
II cherche à répondre à la question: Peut-on justifier au plan moral un jugement, un choix ou une action et comment?

Pour son propos, il réduit la moralité à son expression la plus générale et la plus minimale : les questions de justice. D'abord, cette réduction du domaine de la moralité sert ses ambitions théoriques puisqu'il croit que cette composante constitue le noyau de la moralité et est la plus apte à une saisie par stades structurels "durs". Ensuite, en montrant que les individus ont des capacités morales cognitives qui progressent vers l'acquisition des opérations formelles de justice, et ce pour tous les individus, Kohlberg établit l'existence d'une conception rationnelle minimale de la moralité à laquelle les individus peuvent se référer commeà une base commune pour s'entendre sur ce qui est moral ou non. Cette structure générale et universelle de la moralité s'oppose aux conceptions émotivistes et relativistes.

Les principes formels que la progression à travers les stades nous permet d'acquérir sont l'égalité et la réciprocité, qui se traduisent par les opérations de justice correspondantes, l'universalisabilité et la réversibilité. Ainsi, au niveau préconventionnel de développement (stades 1 et 2 de Kohlberg) mon action est juste si elle satisfait mes besoins et m'évite d'être frappé de sanctions ou punitions. Au niveau conventionnel (stades 3 et 4), la justesse de mon action est évaluée en fonction de sa conformité aux lois et conventions sociales en vigueur. Finalement, au niveau post-conventionnel (stades 5 et 6), mon action n'est considérée juste que si elle repose sur des principes éthiques universaux et valides que je reconnais comme tels.

Au cours de la progression à travers ces stades se développe graduellement une conception de la justice comme impartialité. Cette perspective, appelée aussi «point de vue moral», insiste sur l'importance de parvenir à une entente avec les autres sur ce qui est juste. Cela permet de garantir une certaine impartialité. Cette procédure intersubjective amène la reconnaissance de deux critères pour juger la validité d'un jugement: premièrement, un jugement doit être universalisable; c'est-à-dire qu'il doit 
pouvoir être reconnu par toute personne et appliqué à chacun. Cela implique un second critère : la réversibilité, c'est-à-dire la capacité de se mettre à la place d'autrui et d'adopter son point de vue.

Ces deux éléments sont des qualités formelles appartenant à tout jugement moral valide (indépendamment de son contenu). Ils correspondent aux deux principes généraux que nous acquérons lorsque nous développons un point de vue moral : l'égalité (qui correspond à l'opération d'universalisabilité) et la réciprocité (qui correspond à la réversibilité).

L'égalité et la réciprocité sont les principes les plus fondamentaux communs à tous les jugements moraux indépendamment de leur contenu. Ils définissentune structure rationnelle minimale de la moralité comme justice. Or cette conception minimale formelle est fondamentale puisqu'elle se retrouve à la base de toute conception substantielle valide de moralité dont elle détermine la structure générale. Toutes les théories morales normatives valides doivent référer, selon Kohlberg, d'une façon ou d'une autre, dans la formulation de leurs prescriptions, à des considérations d'égalité et de réciprocité. C'est précisément ce qui garantit que ces normes sont bel et bien «morales».

II faut bien voir que ces principes fondamentaux ne servent, en quelque sorte, qu'à nous assurer de la possibilité même de la moralité et de sa nature et qu'en tant que tel ils ne constituent pas eux-mêmes, du moins sous cette forme, des normes prescrivant une action. Si l'égalité et la réciprocité ont un statut fondamental c'est qu'elles sont inévitablement construites, progressivement, dès lors qu'un être humain entre en contact avec un autre et ce, dès le plus jeune âge ${ }^{3}$. Le degré de formalité qui est conféré à l'égalité et à la réciprocité est suffisant pour

3. Pour Kohlberg le développement moral est incontournable, mais s'il ne se réalise pas au même degré chez tous les individus, il a toujours lieu suivant le modèle dont il rend compte et la progression vers le "point de vue moral" permet toujours d'acquérir une maîtrise plus approfondie des caractéristiques formelles d'égalité et de réciprocité. Ainsi présentée, cette position semble hautement discutable mais il nous faut rappeler qu'elle possède des justifications sérieuses dont la présentation dépasserait le cadre du présent article. Notons simplement que les réflexions de Jürgen Habermas à l'endroit de la théorie de Kohlberg sont particulièrement éclairantes sur ce point. 
délimiter la structure universelle grâce à laquelle il est possible de s'entendre sur la validité d'un jugement moral. En effet, les qualités formelles de justice (l'égalité et la réciprocité) seront opérationnelles pour toute considération morale. Elles peuvent donc être traduites à l'aide de divers principes substantiels qui permettront de définir plus précisément les règles qui doivent guider nos actions afin que celles-ci soient en accord avec ces principes fondamentaux. ${ }^{5}$

Or, ces conceptions substantielles s'attachent à déterminer un contenu à la moralité plutôt qu'à établir formellement ses conditions de possibilité. Leur élaboration présuppose, par ailleurs, qu'on ait clarifié préalablement les questions relatives à l'existence et à la nature de la moralité. La réponse qu'on fournit à la question "comment être moral?» constitue en fait une explication possible de la conception minimale formelle de la moralité qu'une étude métaéthique qui s'est penchée quant à elle sur les questions "qu'est-ce que la moralité?» et «peuton justifier moralement une action? " nous permet de dégager.

À notre avis, les travaux de Gilligan portent sur cet aspect et elle s'intéresse davantage au contenu qu'à la forme de la moralité. Bien qu'elle ne développe pas une théorie morale à proprement parler, les considérations qu'elle met de l'avant visent à mettre en évidence certains éléments susceptibles de guider, dans la pratique, nos actions et jugements moraux. Gilligan cherche aussi à s'opposer à la réduction, trop fréquemment opérée, de la moralité à des considérations de justice formulées en termes de droits et revendications et traduites par les devoirs négatifs (negative duties).

Cela dit, le développement formel dont Kohlberg fait état ne nous fournit pas de principes moraux substantiels comme tels, il ne fait que situer les limites structurelles assurant leur validité. À ce titre, le "point de vue moral" est incapable de nous indiquer lequel de deux principes substantiels valides est le meilleur ou le plus adapté à une situation précise. Ce sont précisément les données (morales ou non) définissant un contexte particulier qui informeront ce choix.

5. Kohlberg reconnaît d'ailleurs ce fait. II ne croit nullement que la moralité se réduit exclusivement aux considérations formelles quil'occupent (voir la note précédente). 
Selon cette tendance réductrice adoptée par plusieurs théories morales normatives, la solution d'un conflit moral consisterait à rétablir un équilibre entre les droits conférés à chacun et les devoirs correspondants. Ainsi, la résolution d'un conflit sera conforme à l'éthique en autant qu'elle ne prive pas autrui de ses droits ou qu'elle n'interfère pas avec ceux-ci. La médiation opérée vise à établir si les droits d'une personne ont été lésés par une autre personne qui aurait failli à ses devoirs et, le cas échéant, à décider d'une réparation satisfaisante et juste pour les deux parties.

Pour Gilligan, ce modèle prédominant d'apparence légale où un conflit dans une situation concrète est résolu par un équilibre des droits et revendications tend à perdre de vue les caractéristiques spécifiques à la situation et à laisser de côté de nombreux éléments déterminants et pertinents au dilemme. Les problèmes moraux se posent dans des contextes où les relations interpersonnelles ne se présentent pas seulement en termes d'équité et de non-interférence mais où elles sont aussi perçues en termes de liens, d'attachement, de rapports aux autres et de sensibilité à leurs besoins. Cette vision alternative (retrouvée principalement chez les femmes, mais pas exclusive à elles) conçoit la solution à des problèmes moraux non pas premièrement en termes d'équilibre entre les parties mais en termes de réponses à l'autre, à ses demandes et à ses besoins, ce qui commande des actions positives visant à prendre soin d'eux et à les aider, préservant ainsi les liens interpersonnels existants.

Gilligan traduit cette préoccupation par un effort conscient pour démontrer une plus grande sollicitude (caring) à l'endroit d'autrui. Elle considère que cette éthique de la sollicitude est complémentaire de l'autre orientation (puisqu'elle rend compte de valeurs importantes qui guident l'action morale). Pour Gilligan, la moralité ne peut se concevoir sans un sentiment d'obligation concrète qui vise à entretenir les liens entre les personnes et à répondre directement aux besoins d'autrui. 
Vus sous l'angle de la sollicitude, les dilemmes moraux sont problématiques dans la mesure où ils font ressortir la difficulté de parvenir à une solution qui ne fasse violence à aucune des parties en présence. Les limites de toute résolution particulière se posent en termes des conflits qui persistent et de leur potentiel à causer du tort (hurt) à autrui. C'est d'ailleurs lorsqu'on se heurte à ces limites qu'apparaît la nécessité d'une médiation qui doit s'opérer dans la gestion des rapports qui nous unissent à autrui et dans la dynamique de nos interactions. C'est par cet exercice qu'il devient possible de développer la capacité de concevoir et de manifester de la sollicitude.

D'ailleurs, une étude menée par Gilligan auprès de vingtneuf femmes devant décider de se faire avorter ou non permet de dégager une séquence de développement de la sollicitude. Cette séquence se caractérise par une réorganisation et un accroissement de l'équilibre dans nos relations interpersonnelles, par une modification du sentiment de responsabilité qui y est lié et de la sollicitude qui en découle. Ainsi, sil'individu se préoccupe d'abord de lui-même pour assurer sa survie dans un monde qu'il juge menaçant, il délaisse bientôt cette attitude égocentrique jugée inacceptable par lui-même et ses pairs en raison de son caractère égoïste. Voyant son comportement ainsi condamné, il comprend que pour être accepté et entrer en relation avec autrui il doit se conformer aux attentes que l'autre entretient à son égard. Son comportement est alors motivé par un sentiment de responsabilité qui l'oblige à combler les besoins d'autrui, en particulier des plus faibles. Le déséquilibre qui résulte de l'équation "sacrifice de soi = sollicitude envers autrui», perçue au départ comme une condition des rapports humains, devient l'élément déclencheur de la seconde transition où seront dénouées les tensions entre égoïsme et responsabilité. La sollicitude devientalors un principe choisi pour guider le jugement orienté en fonction du maintien des liens interpersonnels et de la réponse aux autres, principe qui a pour résultat de condamner en même temps la souffrance, l'abnégation et le sacrifice de soi chez tout être humain. L'obligation de sollicitude se présente alors comme une injonction universelle qui s'étend tant à soi 
qu'aux autres car la médiation qui s'est opérée entre responsabilité et égoïsme a permis de reconnaître l'égalité morale existant entre tous les êtres humains.

Cette brève description de l'éthique de la sollicitude suffit pour nous rendre compte que l'intention qui anime Gilligan est de développer une position substantielle de la moralité pour pallier les lacunes des théories substantielles de justice définissant des principes exprimables seulement en termes de devoirs et de revendications. Son souci principal est de démontrer que la résolution des dilemmes moraux doit toujours tenir compte des éléments particuliers constitutifs d'un contexte donné, ce qui ne serait pas le cas dans les modèles de justice préoccupés par les droits individuels et leur protection. En fait, les différences entre chacun des ces deux modèles peuvent être comprises à l'aide d'une distinction bien connue entre devoirs positifs (positive duties) et devoirs négatifs (negative duties) 5 .

Les devoirs négatifs constituent des interdictions du genre "Tu ne tueras pas». Ces maximes nous commandentl'abstention et ce faisant, contribuent à établir les limites qui permettent de qualifier un comportement de moral ou non. Les devoirs positifs se présentent sous forme de maximes d'action, par exemple "Sois charitable». Elles ne prescrivent pas des actions spécifiques mais délimitent un type de comportement à favoriser. À l'instar des premiers, les devoirs positifs ne sont pas formulés de façon à spécifier leurs limites, leurs domaines et leur degré d'application.

L'éthique de la sollicitude de Gilligan nous renvoie bien à des devoirs positifs. Elle insiste avec raison sur le fait que la moralité ne doit pas être réduite à des principes définissant des droits et devoirs valides absolument. En ce sens,la position qu'elle développe constitue une contribution normative importante, d'autant plus qu'on néglige souvent l'aspect des devoirs

5. Cette distinction est reprise dans l'examen de la controverse Kohlberg/Gilligan par Gertrud Nunner-Winkler, "Two Moralities? A critical discussion of an ethics of care and responsability versus an ethics of rights and justice", In : W.M. Kurtines \& J.L. Gewirtz (éd.), Morality, moral behavior, and moral development. New York, John Wiley and Sons, 1984, p. 348-361. 
positifs. Toutefois, les devoirs positifs ne sont pas les seuls à requérirune sensibilité au contexte pour leur application concrète. Les devoirs négatifs sont rarement parfaits, en ce qu'ils admettent souvent des exceptions qui permettent de transgresser la règle ${ }^{7}$. II n'est donc pas indiqué de les opposer aux devoirs positifs sur cette base comme Gilligan le fait. Plus important encore, ces deux types de devoirs peuvent contribuer ensemble, chacun selon ses particularités, à développer le contenu d'une conception formelle de la moralité. II nous semble maintenant clair que l'effort accompli par Gilligan s'inscrit dans cette veine.

Ce que Gilligan propose est une articulation substantielle des principes formels d'égalité et de réciprocité identifiés par Kohlberg comme composantes formelles de la moralité. Le plaidoyer en faveur de la pratique de la sollicitude trouve sa source dans l'adoption du "point de vue moral» où les êtres humains sont égaux en tant qu'individus et méritent, dès lors, le respect. La dernière étape dans la séquence de développement de la sollicitude atteint précisément ce point. Les opérations de justice (l'universalisabilité et la réversibilité) se concrétisent sous la forme du principe choisi de la sollicitude qui émerge après la résolution des tensions entre responsabilité et égoïsme.

Loin de s'opposer, les modèles de Kohlberg et de Gilligan sont complémentaires. D'un côté, l'éthique de la sollicitude de Gilligan offre une façon d'articuler le modèle formel de la moralité de Kohlberg et de fournir un contenu substantiel à ses principes formels d'égalité et de réciprocité, et de l'autre, la théorie de Kohlberg permet d'apporter une justification rationnelle à l'éthique de la sollicitude de Gilligan.

Les deux positions appartiennent en quelque sorte au même édifice de l'éthique. Chacune y représente un étage, métaéthique pour l'une, normatif pour l'autre et y accomplit des fonctions spécifiques, tantôt justificatives et formelles, tantôt substantielles et normatives, contribuant au maintien de l'édifice de la moralité.

7. Ibid., p. 351-352. Par exemple, la règle "Tu ne tueras pas" peut ne pas valoir dans un cas de légitime défense où un individu doit tuer pour préserver sa propre vie. 
Cette brève réflexion sur les théories de Kohlberg et de Gilligan nous a permis d'étudier la nature de la controverse qui règne entre leurs positions. En situant chacune des entreprises par rapportau type de questionnement éthique qui la préoccupe, nous avons constaté qu'elles possèdent, chacune, un objet propre qui participe néanmoins d'un même projet moral. Ainsi, l'opposition, telle qu'elle est traditionnellement présentée, se fonde sur une conception erronée de la problématique générale abordée par les deux auteurs.

La mise en lumière des défaillances inhérentes à cette opposition traditionnelle nous a permis de présenter une conception alternative originale des liens existant entre la théorie du développement du raisonnement de justice de Kohlberg et l'éthique de la sollicitude de Gilligan et de désamorcer une pseudo-controverse. Cet exercice indique une voie intéressante à explorer dans l'étude de ces auteurs. Cette réorientation ne neutralise certes pas toutes les possibilités de débats et de désaccords mais elle propose une direction fructueuse et novatrice pour reconsidérer l'apport de ces deux auteurs à la clarification du vaste et obscur domaine de la moralité.

\author{
Nathalie Savard \\ Département de philosophie \\ Université du Québec à Trois-Rivières
}




\section{BIBLIOGRAPHIE}

Benhabib, Seyla. "The Generalized and the Concrete Other - The Kohlberg-Gilligan Controversy and Moral Theory”. In : E.F. Kittay \& D.T. Meyers (éd). Women and Moral Theory. Totawa, Rowan \& Littlefields, 1987, p. 154-177.

Blum, Lawrence A.. "Gilligan and Kohlberg: Implications for Moral Theory". Ethics, no. 98 (avril 1988), p. 472-491.

Flanagan, Owen et Jackson, Kathryn. "Justice, Care and Gender : The KohlbergGilligan Debate Revisited". Ethics, no. 97 (avril 1987), p. 622-637.

Flanagan, Owen. "Virtue, Sex and Gender : Some Philosophical Reflections on the Moral Psychology Debate». Ethics, no. 92 (avril 1982), p. 499-512.

Gilligan, Carol. "Do the Social Sciences Have an Adequate Theory of Moral Development». In : N. Haan, R.N. Bellah, P. Rabinow, W.M. Sullivan (éd). Social Science as Moral Inquiry. New York, Columbia University Press, 1983, p. 33-51.

Gilligan, Carol. In a Different Voice - Psychological Theory and Women's Development. Cambridge et Londres : Harvard University Press, 32ème édition, 1993. [En français : Une si grande différence (traduction de A. Kwiatek). Paris, Flammarion, 1986.]

Gilligan, Carol. «Moral Orientation and Moral Development». In : E.F. Kittay \& D.T. Meyers (éd). Women and Moral Theory. Totawa, Rowan \& Littlefields, 1987, p. 1933.

Habermas, Jürgen. Morale et communication (trad. C. Bouchindhomme). Paris, Ed. du Cerf, 1986.

Habermas, Jürgen. De l'éthique de la discussion (trad. M. Hunyadi). Paris, Ed. du Cerf, 1992.

Kerber, L.N., Greeno, C.G., Maccoby, E.E., Luria, Z. Stack, C.B. et Gilligan, C.. "On In a Different Voice : An Interdisciplinary Forum". Signs : Journal of Women in Culture and Society, vol. 11, no. 2, 1986, p. 304-333.

Kohlberg, Lawrence. "A Reply to Owen Flanagan and Some Comments on the PukaGoodpaster exchange». Ethics, no.92 (avril 1982), p. 513-528.

Kohlberg, Lawrence. "From Is to Ought: How to Commit the Naturalistic Fallacy and Get Away with It in the Study of Moral Development", In : Mischel, T. (éd). Cognitive Development and Epistemology. New York et Londres, Academic press, 1971, p. 151-235.

Kohlberg, Lawrence. "The Claim to Moral Adequacy of a Highest Stage of Moral Judgment». The Journal of Philosophy, 70, 1973, p. 630-646.

Kohlberg, Lawrence. The Psychology of Moral Development - the Nature and Validity of Moral Stages (Essays on Moral Development, Vol. II). San Francisco, Harper \& Row Publishers, 1984.

Modgil, S. \& Modgil, C. (éd). Lawrence Kohlberg : Consensus and Controversy. Philadelphie et Londres, The Falmer Press, coll. «Falmer International MasterMinds Challenged", 1985.

Nunner-Winkler, Gertrud. "Two Moralities? A Critical Discussion of an Ethics of Care and Responsability versus an Ethics of Rights and Justice". In : W.M. Kurtines \& J.L. Gewirtz (éd.), Morality, Moral Behavior, and Moral Development. New York : John Wiley and Sons, 1984, p. 348-361. 